\title{
Alarming rise in cervical cytological abnormalities in women living in Jordan
}

\section{Bishtawi ${ }^{1}$, H. Saleh ${ }^{2}$ \\ Al Ahli Hospital, Obstetrics and Gynecology, Doha, Qatar. 2Sidra medicine, Obstetrics and Gynecology, Doha, Qatar.}

\section{Objectives:}

Cervical cancer is preceded by a group of epithelial cell abnormalities. However, there is insufficient data on cervical abnormalities in Jordan and the Middle East at large. The current study aimed at determining the prevalence of different cytological abnormalities in women in Jordan. In addition, it aimed at assessing the age specific cytological abnormalities in these women and analyzing the changing trends of epithelial cell abnormalities in cervical smear over a period of 15 years compared in three periods of five years each.

Methods:

6,455 conventional cervical Papanicolaou (Pap) smear results obtained between January 2000 and December 2014 were retrospectively analyzed. Results: Out of the 6,454 Pap smears analyzed, 5,645 (87.5\%) were found adequate for reporting.

\section{Results}

A total number of 801 (14.2 \%) cases had cervical epithelial abnormalities. A significant increase in cytological abnormalities was observed between 2000 and 2014. In addition, a significant increasing trend in cervical cytological abnormalities was noted between 2000 and 2014. The highest percentage of cytological abnormalities (20.1\%) was found in women younger than 25 years old. In all of the age groups, the low-grade squamous intraepithelial lesions (LSIL) cytological abnormality was the most prevalent

\section{Conclusions:}

Invasivecervical cancer is still a killer for young women in the developing countries. The present study may reflect a change in the sociosexual behavior over the last 15 years. The current work highlights the importance of awareness campaigns on the importance of cervical smear and the urgent need for initiating a cervical screening program in Jordan.

\begin{tabular}{|c|c|c|c|c|}
\hline Cytological smear result & $\begin{array}{c}\text { Total } \\
\text { 2000-2014 }\end{array}$ & $\begin{array}{c}\text { Period I } \\
\text { 2000-2004 }\end{array}$ & $\begin{array}{c}\text { Period 2 } \\
\text { 2005-2009 }\end{array}$ & $\begin{array}{c}\text { Period 3 } \\
\text { 2010-2014 }\end{array}$ \\
\hline \multirow[t]{2}{*}{ Normal } & 4,825 & 563 & 2,341 & 1,921 \\
\hline & (85.5) & $(93.8) \stackrel{2,3,63}{=}$ & $(87.9)^{c 3}$ & $(80.6)$ \\
\hline \multirow[t]{2}{*}{ Ascus } & 204 & 4 & 98 & 102 \\
\hline & (3.6) & $\left(\underline{0.6)^{3,3, b 3}}\right.$ & (3.7) & (4.2) \\
\hline \multirow[t]{2}{*}{ AGUS } & 90 & 11 & 29 & 50 \\
\hline & (1.6) & (1.8) & $(1.1)^{c^{2}}$ & (2.1) \\
\hline \multirow[t]{2}{*}{ LSIL } & 463 & 16 & 153 & 294 \\
\hline & (8.2) & 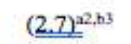 & $(5.7)^{3}$ & (12.3) \\
\hline \multirow[t]{2}{*}{ HSIL } & 42 & 4 & 30 & 8 \\
\hline & (0.7) & (0.7) & $(1.1)^{c 2}$ & (0.3) \\
\hline \multirow{2}{*}{ Carcinoma } & 2 & 0 & 0 & 2 \\
\hline & $(0.04)$ & $(0.0)$ & $(0.0)$ & $(0.1)$ \\
\hline \multirow[t]{2}{*}{ Others } & 19 & 2 & 12 & 5 \\
\hline & (0.3) & (0.3) & (0.5) & $(0.2)$ \\
\hline Total & 5,645 & 600 & 2,663 & 2,282 \\
\hline
\end{tabular}

$\mathbf{a}^{2}: \mathbf{p}<0.01 \mathbf{a}^{3}: \mathbf{p}<0.001$ period 1 versus period $2 ; \mathbf{b}^{3}: \mathbf{p}<0.001$ period 1 versus period $3 ; \mathbf{c}^{2}: \mathbf{p}<$

$0.01, \mathrm{e}^{3}: \mathrm{p}<0.001$ period 2 versus period 3 .

\begin{tabular}{|c|c|c|c|c|c|}
\hline $\begin{array}{l}\text { Cytological smear } \\
\text { result }\end{array}$ & Total & $<25$ years & $25-35$ years & 36-45 years & $>45$ years \\
\hline \multirow[t]{2}{*}{ Normal } & 3,994 & 126 & 912 & 1,389 & 1,567 \\
\hline & (85.6) & (79.99 (a,b & (85.2) & (85.8) & $(86.2)$ \\
\hline \multirow[t]{2}{*}{ Ascus } & 157 & 7 & 35 & 60 & 55 \\
\hline & (3.4) & (4.4) & (3.3) & (3.7) & (3.0) \\
\hline \multirow[t]{2}{*}{ AGUS } & 79 & 3 & 11 & 27 & 38 \\
\hline & (1.7) & (1.9) & (1.0) & (1.7) & (2.1) \\
\hline \multirow[t]{2}{*}{ LSIL } & 383 & 21 & 106 & 126 & 130 \\
\hline & $(8.2)$ & $\left(\underline{13,3)^{2,22}}\right.$ & $\left(\underline{2.9)^{2}}\right.$ & (7.7) & (7.2) \\
\hline \multirow[t]{2}{*}{ HSIL } & 35 & 1 & 5 & 11 & 18 \\
\hline & $(0.8)$ & $(0.6)$ & $(0.5)$ & $(0.8)$ & (1.0) \\
\hline \multirow[t]{2}{*}{ Carcinoma } & 2 & 0 & 0 & 1 & 1 \\
\hline & $(0.04)$ & $(0.0)$ & $(0.0)$ & $(0.06)$ & $(0.05)$ \\
\hline \multirow[t]{2}{*}{ Others } & 16 & 0 & 2 & 5 & 9 \\
\hline & $(0.3)$ & $(0.0)$ & $(0.2)$ & $(0.3)$ & $(0.5)$ \\
\hline Total & 4,666 & 158 & 1,071 & 1,619 & 1,818 \\
\hline
\end{tabular}

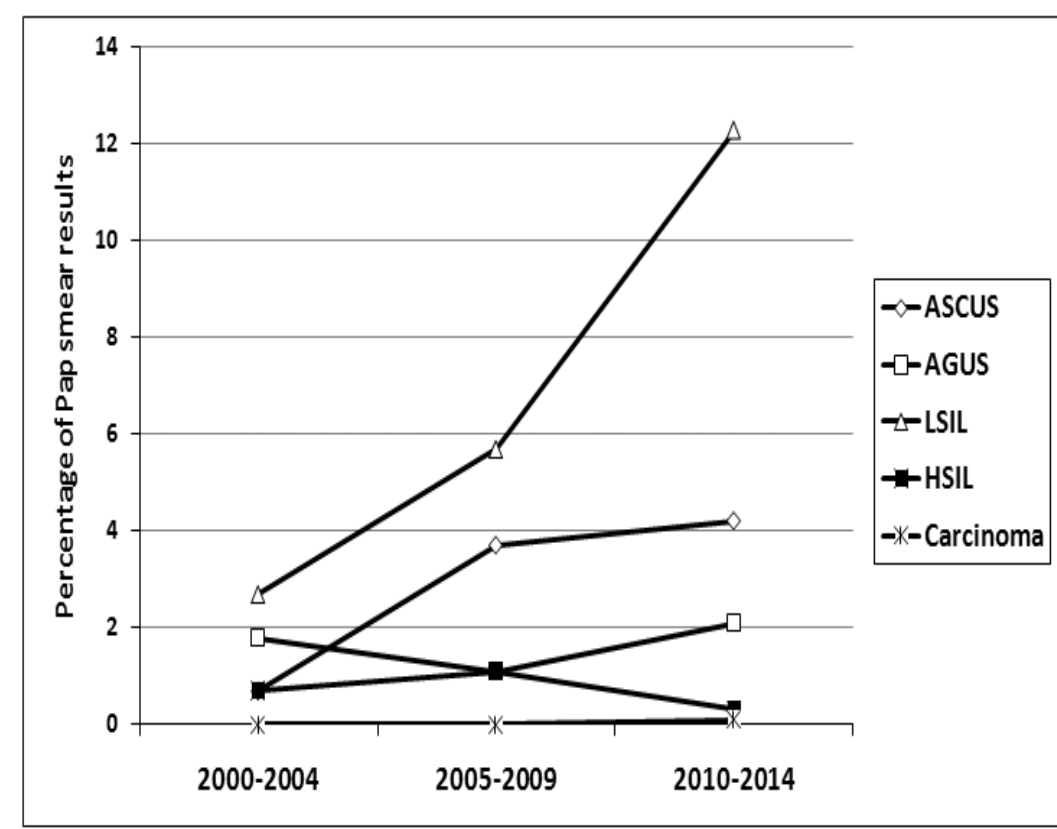

Figure 2.

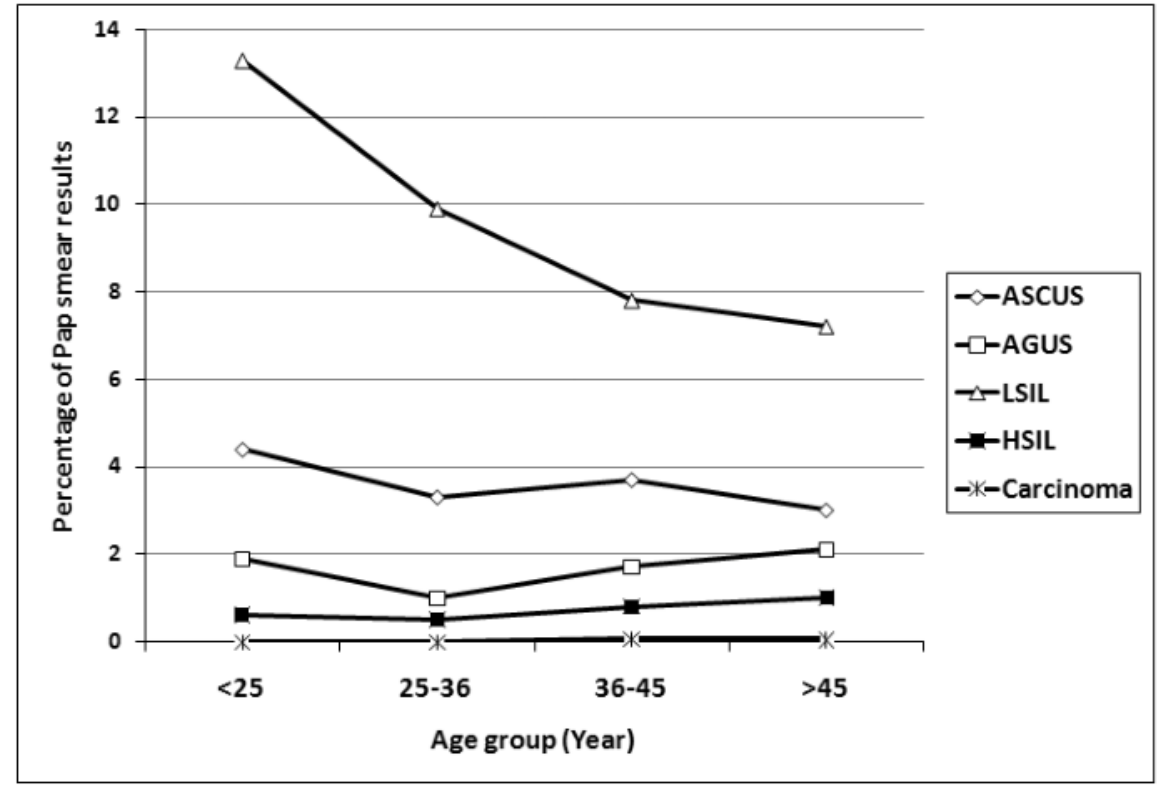

a: $p<0.05$ age group 1 versus age group $3 ; b: p<0.05, b^{2} p<0.01$ age group 1 versus age group

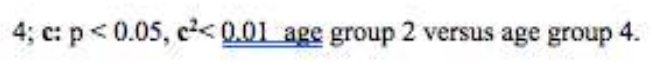

